\title{
Qatar Biobank Milestones in Building a Successful Biobank
}

\author{
Ayat Salman, ${ }^{1-3,{ }^{*}}$ Ronny Baber, ${ }^{3-5,{ }^{*}}$ Linda Hannigan, ${ }^{6}$ Jens Karsten Habermann, ${ }^{3,7,8}$ \\ Marianne K. Henderson, ${ }^{9,10}$ Michaela Th. Mayrhofer, ${ }^{11}$ and Nahla Afifi ${ }^{6}$
}

$\mathrm{Q}$ ATAR BIOBANK (QBB), a center within Qatar Foundation, was established in collaboration with Hamad Medical Corporation and the Ministry of Public Health to enable local scientists to conduct medical research on prevalent health issues in Qatar. Since its establishment in 2012, ${ }^{1}$ QBB has registered >23,000 Qatari participants within a pilot phase that ended in 2016. Today, QBB is one of the leading clinical biobank innovators supporting population and genetic research for personalized medicine in biobanking science and research in the Gulf and Middle East regions and beyond. An example of such innovation includes the design and creation of a Qatari Chip, ${ }^{2}$ which is validated for use in research and is in the process of accreditation for diagnostic purposes.

This Biopreservation and Biobanking special section features a comprehensive overview of QBB's strategic framework for optimal utilization of resources, infrastructure, governance, and management structures dedicated to bringing together multidisciplinary stakeholders to translate biobank science into evidence-based health care interventions. Moreover, this special section includes the details of the QBB's information technology infrastructure to support its mission. QBB implemented an integrated management system (IMS) that incorporates ISO 9001:2015 and ISO 27001:2013 standards. The IMS ensures that rigorous processes and controls are in place, not only to manage the quality of internal and external processes and services provided, but also to maintain the privacy and confidentiality of data collected during participant visits. The approach of data integration, methods for data extraction, de-identification, and the consolidation of data in a shared data model, as well as the major challenges to build and maintain such an infrastructure, are also described.

A special meeting report is included from The International Biobanking Conference entitled "Quality Matters: A Global Discussion in Qatar,', which was held on March 25-27, 2019, in Doha, Qatar. The 3-day event was organized and hosted by QBB and the European, Middle Eastern, and African Society for Biopreservation and Biobanking (ESBB), with supporting collaboration from the International Society for Biological and Environmental Repositories (ISBER) and the Biobanking and BioMolecular Resources Research Infrastructure-European Research Infrastructure Consortium (BBMRI-ERIC). The scientific agenda drew $>1000$ scientists, researchers, industry experts, and health professionals from five continents. Researchers, scientists, and experts from around the world were invited to present along with special presentations from QBB, showcasing their standing as a leading clinical biobank innovator in support of population and genomic medicine. Also, in this special section, QBB highlights some of the information technology featured at the biobank that allows for ongoing advances in research, national and international collaborations, as well as processes to maintain high quality standards and achievements. In this special section, QBB describes their path toward the implementation of best practices in their daily biobanking activities, as well as the next steps toward standardization in the biobank, including ongoing discussions in the area of best practices and standards. This is a milestone for QBB to achieve best practices in collecting, processing, storing, and finally sharing

${ }^{1}$ McGill University Health Center, Royal Victoria Hospital (Glen), Quebec, Canada.

${ }^{2}$ Department of Family Medicine, McGill University, Quebec, Canada.

${ }^{3}$ European, Middle Eastern and African Society for Biopreservation and Biobanking (ESBB), Brussels, Belgium.

${ }^{4}$ Institute of Laboratory Medicine, Clinical Chemistry and Molecular Diagnostics, University of Leipzig Medical Center, Leipzig, Germany.

${ }^{5}$ Leipzig Medical Biobank, University Leipzig, Leipzig, Germany.

${ }^{6}$ Qatar Biobank, Qatar Foundation, Doha, Qatar.

${ }^{7}$ Interdisciplinary Center for Biobanking-Lübeck (ICB-L), University of Lübeck, Lübeck, Germany.

${ }^{8}$ Section for Translational Surgical Oncology and Biobanking, Department of Surgery, University of Lübeck and University Hospital Schleswig-Holstein, Lübeck, Germany.

${ }^{9}$ National Cancer Institute, National Institutes of Health, DHHS, Bethesda, Maryland.

${ }^{10}$ International Society for Biological and Environmental Repositories (ISBER), Vancouver, Canada.

${ }^{11}$ Biobanking and BioMolecular Resources Research Infrastructure-European Research Infrastructure Consortium (BBMRI-ERIC), Graz, Austria.

*Co-first authors. 
high-quality biological samples and associated data for research purposes.

\section{References}

1. Qatar Biobank. www.qatarbiobank.org.qa (accessed October 29, 2019).

2. Qatar Genome. Qatar Genome and Qatar Biobank Lead Efforts to Produce First Qatari Gene Chip. https://qatargenome .org.qa/node/138 (accessed October 29, 2019).
Address correspondence to: Nahla Afifi, MD, PhD Qatar Biobank Qatar Foundation

Hamad Medical City, Building 317

P.O. Box 5825

Doha 9744

Qatar

E-mail: nafifi@qf.org.qa 